\title{
Mineração
}

\section{Composição química de agregados mistos de resíduos de construção e demolição do Estado de São Paulo}

\author{
(Chemical composition of mixed construction and demolition recycled \\ aggregates from the State of São Paulo)
}

Carina Ulsen

Pesquisadora do LCT-EPUSP, Doutoranda em Engenharia Mineral Depart. de Engenharia de Minas e de Petróleo Escola Politécnica da USP E-mail: carina.ulsen@poli.usp.br

Henrique Kahn

Prof. Dr. Associado Depart. de Engenharia de Minas e de Petróleo da Escola Politécnica da USP

E-mail: henrique.kahn @poli.usp.br

\section{Sérgio C. Angulo}

Pesquisador. Instituto de Pesquisas Tecnológicas do Estado de São Paulo E-mail:scangulo@ipt.br

\section{Vanderley M. John}

Prof. Dr. Associado Depart. de Engenharia de Construção Civil da Escola Politécnica da USP E-mail:vanderley.john@poli.usp.br

\section{Resumo}

Os Resíduos de Construção e Demolição (RCDs) representam cerca de 50\% dos resíduos sólidos de cidades brasileiras; a reciclagem é, portanto, desejável, tanto do ponto de vista econômico, como ambiental. Nesse trabalho são apresentados resultados de estudos de caracterização química referentes a amostras de duas usinas de reciclagem do Estado de São Paulo. Verificou-se que a classificação corrente do RCD, segundo critérios visuais (tipos cinza e vermelho), não está relacionada à composição química destes, sendo esta influenciada, principalmente, pela granulometria. A composição química dos RCDs varia em função dos conteúdos de pasta de cimento, tipos dos agregados naturais (areia quartzosa ou granito), cerâmica vermelha e argila. Ainda, para a reciclagem dos resíduos como agregados para concretos, são necessários dois estágios de britagem para atendimento às normas ABNT. A fração miúda apresenta um enriquecimento nos teores de sílica, indicando liberação da pasta de cimento para os finos $(<0,15$ mm). Os finos têm aplicações potenciais na indústria de cimento.

Palavras-chave: Resíduos de construção, caracterização tecnológica, reciclagem.

\section{Abstract}

Construction and Demolition Waste (CDW) represents about 50\% of the total Brazilian municipal solid waste; thus, recycling represents huge benefits both in environmental and economic perspectives. Herein, the chemical characterization results of three samples from two different recycling plants from the State of Sao Paulo is prevented. The results demonstrated that the visual classification into grey and red is not related to the chemical composition but mostly to the grain size fraction. The chemical composition of the CDW varies according to the content of cement paste, natural aggregates (quartz sand or granite), red ceramic and clay. Furthermore, the production of recycled concrete aggregates requires two crushing stages to meet the technical standards. The sand fraction (below $4.8 \mathrm{~mm}$ ) presents high grades of $\mathrm{SiO}_{2}$, which indicates the liberation of cement paste to fines $(<0.15 \mathrm{~mm})$. The fines have a great potential to be used in the cement industry.

Keywords: Construction debris, technological characterization, recycling. 


\section{Introdução}

Os Resíduos de Construção e Demolição (RCDs) são um dos principais responsáveis pelo esgotamento de áreas de aterros em cidades de médio e grande porte, uma vez que eles correspondem a mais de $50 \%$ em massa dos resíduos sólidos urbanos (John, 2000; EC, 2000). No Brasil, estima-se que sejam gerados anualmente cerca de $70 \times 10^{6}$ t/ano de RCDs (Angulo, 2005).

Além disso, dada a ausência de soluções adequadas, uma parcela significativa dos resíduos da construção civil é depositada irregularmente (Pinto, 1999), gerando altos custos sócio-econômicos e ambientais para as administrações municipais. Na cidade de São Paulo, por exemplo, os gastos referentes à coletatransporte-deposição dos RCDs pela prefeitura são da ordem de $\mathrm{R} \$ 45,0 \mathrm{x}$ 10\%/ano (Schneider, 2003).

A composição dos RCDs depende, essencialmente, da natureza dos processos construtivos e, consequentemente, dos materiais empregados. No Brasil e em alguns países europeus, como a Holanda (Hendriks, 2000), os RCDs são constituídos, principalmente, por uma mistura de concreto, argamassa, cerâmica e rochas (Carneiro et al., 2000; Ferraz et al., 2001). Essa fração inorgânica, de origem mineral, é adequada para a reciclagem, atividade fundamental para reduzir os impactos ambientais negativos desses resíduos (John, 2000; EC, 2000; CONAMA, 2002), podendo gerar matéria-prima secundária em substituição à natural, não-renovável e escassa nas proximidades das grandes cidades e em algumas cidades da região amazônica.

Até mesmo na Europa, em que a reciclagem vem sendo realizada desde a segunda guerra mundial (Hendriks, 2000), a principal utilização dos agregados reciclados é voltada para atividades de pavimentação. Nesse caso, os requisitos técnicos são menos exigentes e, geralmente, um circuito de britagem com peneiramento é suficiente para o beneficiamento. Há uma necessidade crescente de utilizá-los em outras aplicações como argamassas, concretos (Hendriks, 2000; Angulo et al., 2002) e matérias-primas industriais (Mueller, 2003; Bianchini et al., 2005).

Os métodos tradicionais de caracterização de RCDs baseiam-se, majoritariamente, em determinar a composição do resíduo, visualmente, em termos da proporção de concreto, argamassa e materiais cerâmicos; porém tal avaliação não permite separar, adequadamente, concreto e argamassa, porque é difícil avaliar o teor presente de pasta de cimento ou, até mesmo, a porosidade da mesma (Angulo, 2005). A composição química dos RCDs pode ser um dos parâmetros decisivos na avaliação da possibilidade de utilização do material reciclado em aplicações diversas. Várias pesquisas demonstram que o teor de pasta de cimento aderida, que pode ser estimada a partir da composição química (Angulo, Mueller, 2007), está associado à porosidade dos agregados reciclados (Nagataki et al., 2004; Linsz, Mueller, 2004). O desenvolvimento de procedimentos de caracterização mais abrangentes é recomendado e necessário para maior controle da qualidade dos produtos gerados e aumento da credibilidade dos agregados reciclados, podendo, inclusive, levar ao diagnóstico de novas aplicações ou inviabilizar as aplicações desejadas. Entre os procedimentos recomendados, consideram-se parâmetros como a composição química (Limbachiya et al., 2006), mineralógica (Bianchini et al., 2005), microestrutural (Nagataki et al., 2004; Linsz; Mueller, 2004) e a densidade (Ulsen, 2006), bem como o desenvolvimento de tecnologias de processamento mais elaboradas, em que a densidade passe a ser um critério relevante (Jungmann, Quindt, 1999).

Nesse trabalho, discute-se, unicamente, a composição química em amostras representativas de agregados de RCDs mistos (com teores variáveis de cerâmica vermelha) provenientes de duas usinas de reciclagem do Estado de São Paulo: Itaquera e Vinhedo.

\subsection{Usinas de reciclagem amostradas}

As usinas de reciclagem classificam os resíduos em duas classes de acordo com a sua cor: cinza, composto, visualmente, por materiais cimentícios, e vermelho, composto, visualmente, por materiais de cerâmica vermelha. Essa classificação, embora realizada sem critério tecnológico, tem, tradicionalmente, aplicações preferenciais distintas: o agregado cinza, em tese, poderia ser utilizado em componentes pré-fabricados à base de cimento (blocos, calçamentos), além de bases de pavimentação, enquanto o vermelho está restrito somente a última aplicação citada.

Os estudos foram realizados sobre três amostras: a) cinza-Itaquera, b) vermelho-Itaquera e c) vermelho-Vinhedo, provenientes de duas diferentes usinas de reciclagem, Itaquera e Vinhedo. Uma descrição sucinta das usinas supracitadas é apresentada a seguir:

a) Usina de Itaquera: localizada na antiga pedreira Itaquera, zona leste da cidade de São Paulo. Processava na ocasião 400 t de RCDs/dia (13\% da capacidade nominal), em que a proporção entre cinza e vermelho é, aproximadamente, a mesma (50\% cada); os resíduos cinza e vermelho eram amostrados separadamente.

b) Usina de Vinhedo, localizada a $100 \mathrm{~km}$ da cidade de São Paulo e processando até $40 \mathrm{t}$ de RCDs/dia (77\% da capacidade nominal), em que somente $25 \%$ do volume do material é classificado como cinza.

A usina de Itaquera empregava as seguintes operações unitárias: seleção manual (ou catação - antes e após a britagem), cominuição em britador de impacto (capacidade de $100 \mathrm{t} / \mathrm{h}$ ), separação magnética após a britagem e peneiramento a seco em peneira inclinada de 3 decks (40, 20 e 4,8 mm). Na usina de Vinhedo, as operações eram: seleção manual para remoção de contaminantes e da fração metálica, cominuição em britador de mandíbulas (capacidade de $8 \mathrm{t} / \mathrm{h}$ ) e peneiramento a seco em 3 decks (12,7; 9,5 e 4,8 mm). 


\section{Materiais e métodos}

O procedimento experimental adotado compreendeu as atividades apresentadas, esquematicamente, na Figura 1 e descritas a seguir.

- Amostragem primária conduzida nas usinas durante 20 dias de produção em intervalos regulares de duas horas, na correia transportadora, após a britagem.

- Homogeneização em pilha alongada e amostragem secundária de todo o material coletado (Petersen, 2005) para retirada de alíquotas de, aproximadamente, 1/6 da massa inicial.

- Homogeneização em pilha de homogeneização alongada seguida de amostragem terciária em laboratório com a retirada de alíquotas para os estudos de caracterização. Os produtos amostrados foram denominados “Tal Qual - TQ” e identificados por: ITC - Itaquera cinza, ITV- Itaquera vermelho e VIV - Vinhedo vermelho.

- Análise granulométrica a úmido do TQ em peneirador mecânico com telas de $50 \times 50 \mathrm{~cm}$ e aberturas nominais de 25,$4 ; 19,1 ; 12,7 ; 9,5 ; 4,8 ; 2,4 ; 1,2$; 0,6; 0,3 e $0,15 \mathrm{~mm}$.

- Britagem das frações retidas em 25,4 mm em britador de mandíbulas 2015, marca Furlan, em circuito fechado com peneiras nas mesmas aberturas até a obtenção de todo o material passante. O produto da britagem secundária foi denominado "produto britado - PB”.

- Peneiramento a úmido do PB em 4,8; 2,4; 1,2; 0,6; 0,3 e 0,15 mm.

- Composição das frações granulométricas do "PB" com a fração abaixo de 25,4 mm do "TQ", denominados finos da britagem primária "FBP”.

- Análises químicas dos produtos obtidos por espectrometria de fluorescência de raios X (FRX) através de espectrômetro Magix Pro, marca PANalytical, em pérolas fundida com determinações sistemáticas de $\mathrm{SiO}_{2}, \mathrm{Fe}_{2} \mathrm{O}_{3}, \mathrm{Al}_{2} \mathrm{O}_{3}, \mathrm{CaO}, \mathrm{MgO}$, $\mathrm{Na}_{2} \mathrm{O}, \mathrm{K}_{2} \mathrm{O}, \mathrm{MnO}, \mathrm{TiO}_{2}, \mathrm{P}_{2} \mathrm{O}_{5}$ e $\mathrm{SO}_{3}$, e perda ao fogo com permanência à temperatura de $1.050^{\circ} \mathrm{C}$ por uma hora. A preparação das amostras, para análises químicas, envolveu procedimentos sucessivos de cominuição em britador de mandíbulas e moinho de rolos seguidos de amostragem em equipamento tipo “Jones” para obtenção de alíquotas representativas, as quais foram pulverizadas em moinho de discos oscilantes previamente às análises.

\section{Discussão dos resultados}

\subsection{Análise granulométrica}

A distribuição granulométrica acumulada na fração passante para os produtos “Tal Qual” (TQ) das três amostras é expressa na Figura 2a. Os agregados de RCDs reciclados de Itaquera (ITC e ITV) apresentam, aproximadamente, $20 \%$ de sua massa acima de $25,4 \mathrm{~mm}$, enquanto que o agregado de Vinhedo (VIV) apresenta 45\%. Essa fração é

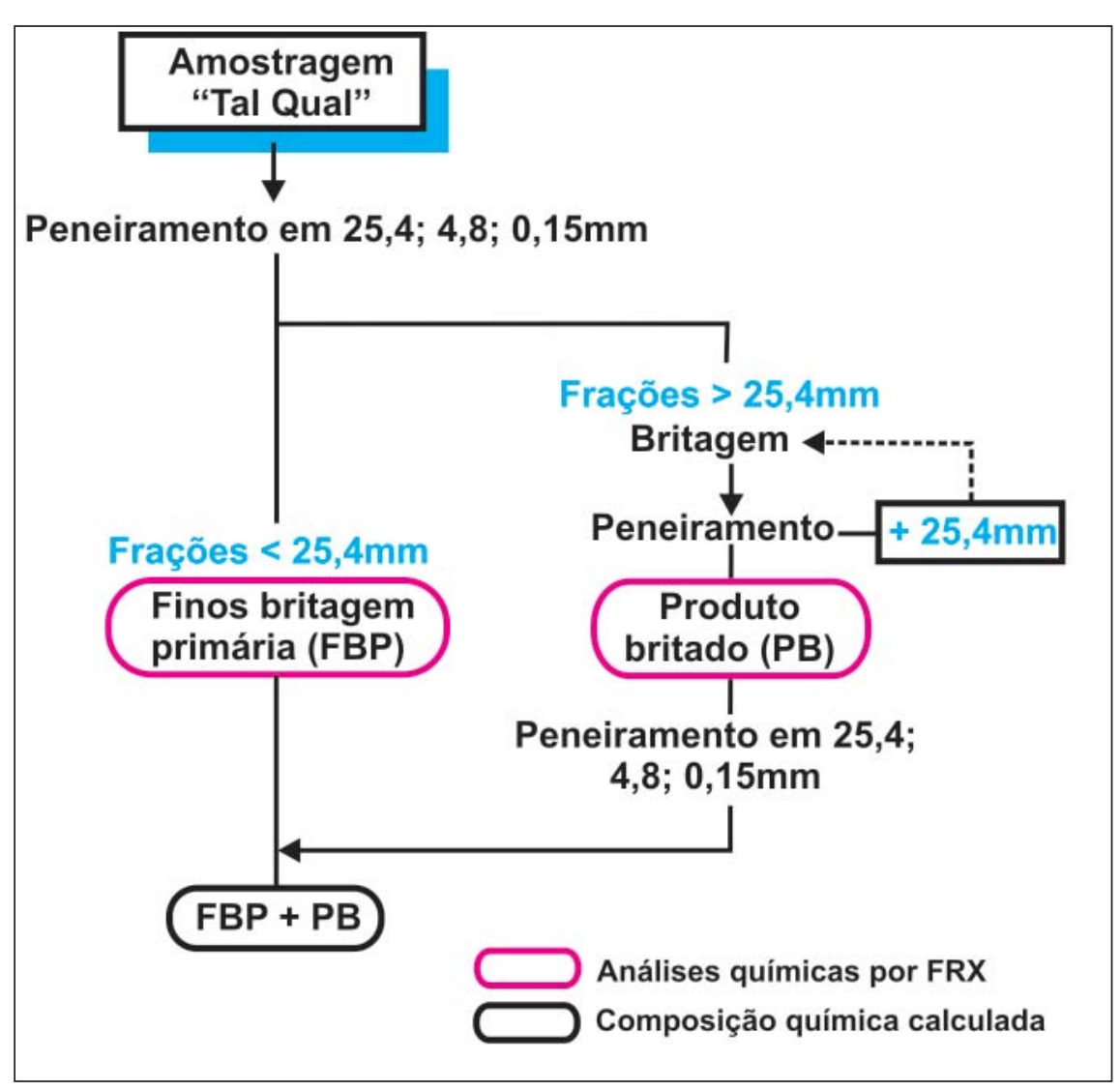

Figura 1 - Procedimento experimental aplicado a RCDs britados. de pouco interesse para o emprego em concretos, já que a dimensão máxima dos agregados graúdos mais utilizada para concreto (brita tipo 1 e 2) é 25,4 mm (NBR NM 248).

A proporção de agregados miúdos (abaixo de 4,8 mm) no RCD chega a cerca de 50\%. Atualmente, não existem aplicações difundidas para essa fração, exceto quando aplicada em base de pavimentação, pois possui elevada porosidade, forma irregular e elevado teor de contaminantes (Rilem Recomendation, 1994). Nesse contexto, há a necessidade do desenvolvimento de tecnologias e novos mercados para essa fração.

A britagem secundária permitiu adequar a granulometria dos agregados reciclados para possível uso em concretos. A distribuição granulométrica apresentada na Figura 2b mostra que o segundo estágio de britagem gera um produto com 70 a $85 \%$ de massa na fração graúda $(-25,4+4,8 \mathrm{~mm})$ e um conteúdo de finos $(-0,15 \mathrm{~mm})$ da ordem de $5 \%$.

REM: R. Esc. Minas, Ouro Preto, 63(2): 339-346, abr. jun. 2010 

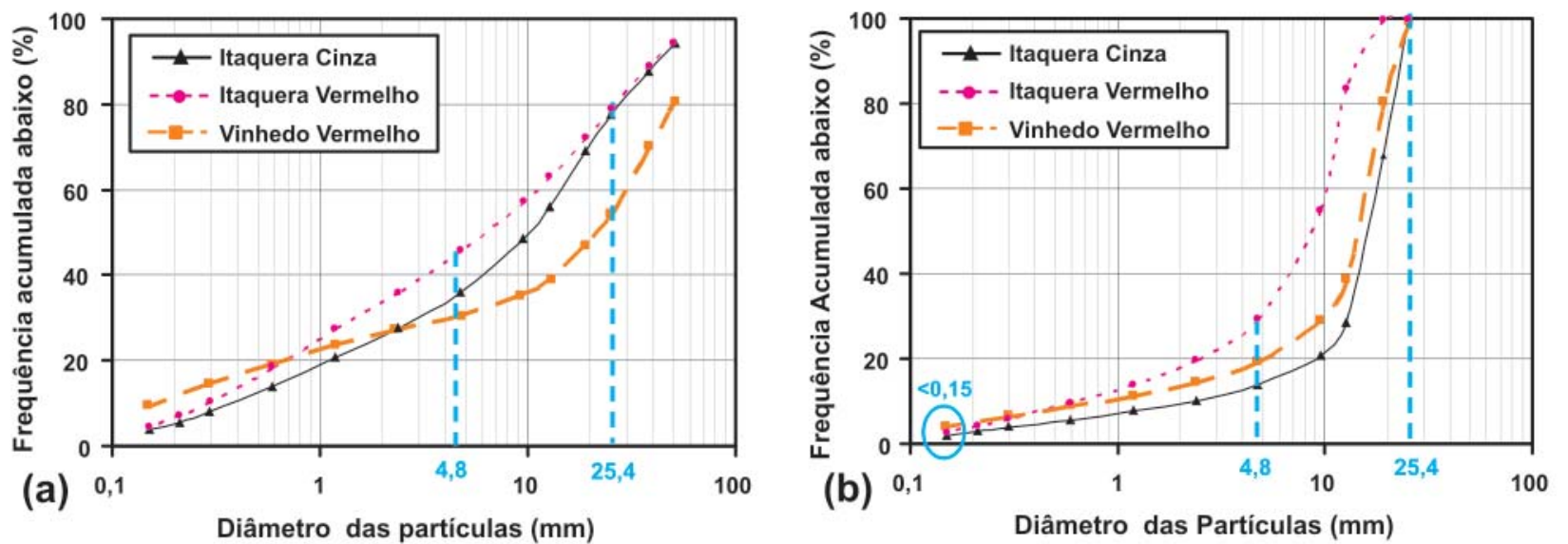

Figura 2 - Distribuição granulométrica dos produtos "Tal Qual - TQ" (a) e dos produtos "Produto Britado - PB" (b).

A Figura 3 apresenta a curva de distribuição granulométrica resultante da composição proporcional do material abaixo de $25,4 \mathrm{~mm}$ (FBP) com o produto da britagem secundária (PB), gerando os seguintes produtos: a) $50-60 \%$ de agregado graúdo, b) $25-45 \%$ de agregado miúdo e c) $5-15 \%$ de finos (abaixo de $0,15 \mathrm{~mm})$.

Adicionalmente, verifica-se que a distribuição granulométrica dos resíduos da classe "vermelho" é sempre mais fina do que a classe de resíduos "cinza", indicando menor resistência das fases cerâmicas e/ou maior quantidade de solo ou material pulverulento na amostra. O mecanismo de britagem primária resulta em uma distribuição granulométrica mais grossa para o RCD de Vinhedo (britador de mandíbulas) com maior conteúdo de finos (características do RCD); essa diferença é neutralizada após britagem secundária.

\subsection{Composição química dos agregados mistos de RCDs}

Os teores médios dos principais óxidos para os produtos graúdo, miúdo e finos são apresentados na Tabela 1; a composição granuloquímica das três amostras compostas (FBP + PB) é apresentada na Tabela 2.

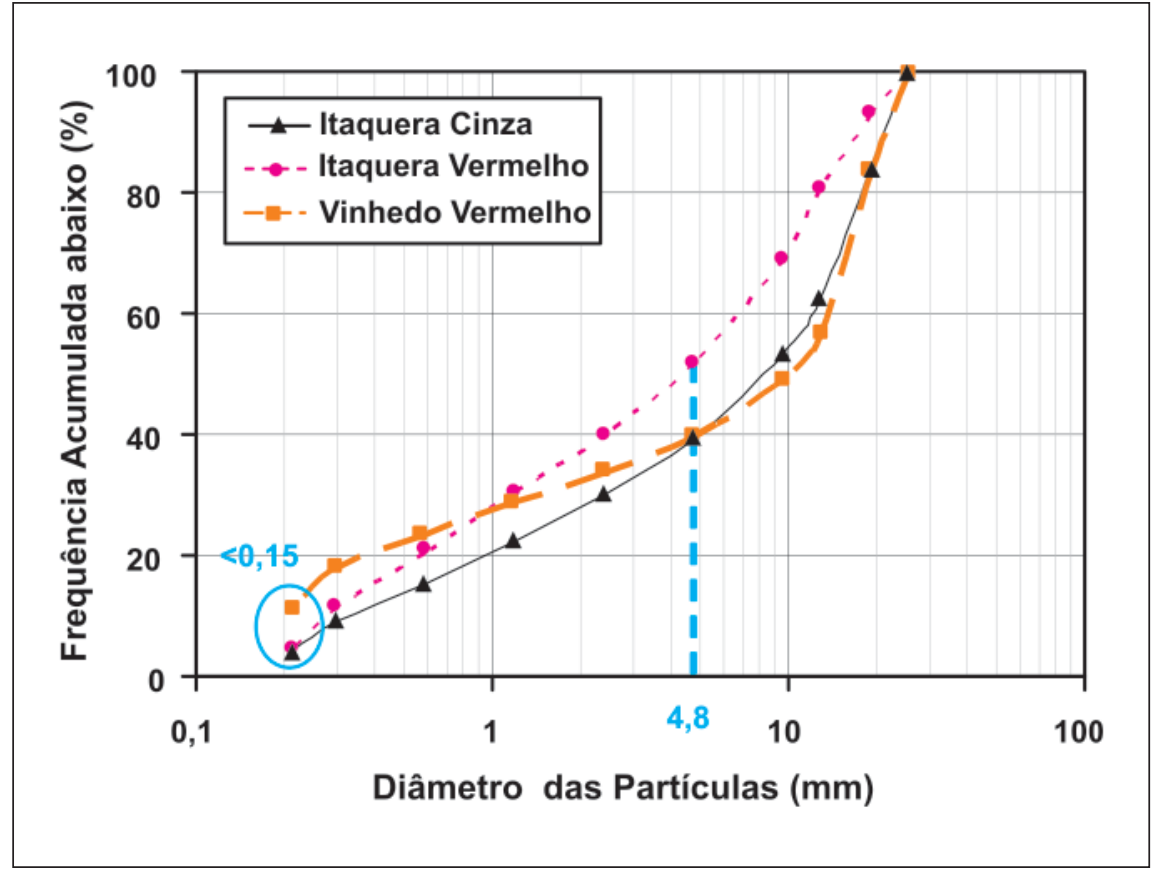

Figura 3 - Distribuição granulométrica do produto composto (FBP + PB).

A sílica $\left(\mathrm{SiO}_{2}\right)$ é o principal óxido constituinte do RCD (Tabela 2), com teores entre 48 e $84 \%$; sua origem está associada, principalmente, aos agregados naturais do concreto e da argamassa presentes no RCD. A alumina $\left(\mathrm{Al}_{2} \mathrm{O}_{3}\right)$ e o óxido de cálcio $(\mathrm{CaO})$ são os outros óxidos mais representativos, com teores entre 4,7 e 11\% e entre 2,4 e $14 \%$, respectivamente. A alumina está relacionada, principalmente, à presença da cerâmica vermelha e do solo e, secundariamente, à presença de feldspatos e do cimento. O óxido de cálcio está associado ao aglomerante (pasta de cimento endurecida, cal, gesso, etc.) presente em componentes construtivos, já que os agregados originais não são de natureza calcária. Tal situação é específica para as amostras estudadas; o mesmo não ocorre para regiões que utilizam agregados de rocha calcária, onde as composições químicas são, significativamente, diferentes. 
Carina Ulsen et al.

A perda ao fogo varia de 3,35 a 19,6\%, estando, essencialmente, relacionada à liberação da água de constituição e gás carbônico dos silicatos de cálcio hidratado, cal hidratada e carbonatos, liberação de água dos filossilicatos provenientes dos solos, cerâmicas queimadas abaixo de $500^{\circ} \mathrm{C}$ e de outros minerais menores presentes na fração de agregado graúdo (brita derivada de rochas como granitos; Klein, Hurburt, 1937). A Figura 4 mostra que a perda ao fogo é proporcional aos teores de $\mathrm{CaO}$ e alumina e inversamente proporcional aos teores de sílica, demonstrando que está relacionada à perda de água e do $\mathrm{CO}_{2}$ do aglomerante, como, também, da argila.

\subsubsection{Influência da granulometria}

A composição química está diretamente relacionada à fração granulométrica das amostras (Figura 5a e Tabela 2), sendo que os teores de $\mathrm{CaO}$ e a perda ao fogo aumentam nos finos $(-0,15 \mathrm{~mm})$, enquanto os de sílica decrescem. Tal composição indica que essa fração apresenta maiores conteúdos de pasta de cimento, cerâmica vermelha ou argila. Além disso, a fração miúda apresenta um enriquecimento nos teores de sílica, associado à redução dos teores de óxido de cálcio e alumina (Figura 4a), indicando liberação da pasta de cimento para os finos $(-0,15 \mathrm{~mm})$ e, portanto, enriquecimento de sílica.

\subsubsection{Influência da britagem secundária}

Os teores médios de $\mathrm{SiO}_{2}, \mathrm{Al}_{2} \mathrm{O}_{3}$ $\mathrm{CaO}$ e PF, por fração granulométrica dos Finos da Britagem Primária (FBP), são muito semelhantes aos teores do Produto Britado (PB). Isso demonstra que não há heterogeneidade de constituição entre a fração acima e abaixo de 25,4 mm (Tabela 1 e Figura 5b). Desta forma, a composição do FBP com o PB aumenta a proporção da fração de agregado graúdo para concreto, sem implicar maiores alterações na composição química desse produto.

Tabela 1 - Sumário da composição granuloquímica dos Finos da Britagem Primária (FBP) e do Produto Britado (PB).

\begin{tabular}{|c|c|c|c|c|c|c|c|c|}
\hline & \multirow{2}{*}{ Produtos } & \multicolumn{2}{|c|}{ Itaquera cinza } & \multicolumn{2}{|c|}{ Itaquera vermelho } & \multicolumn{2}{|c|}{ Vinhedo vermelho } \\
\hline & & & FBP & PB & FBP & PB & FBP & PB \\
\hline \multirow{3}{*}{\multicolumn{2}{|c|}{ 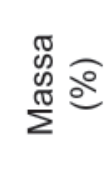 }} & Graúdo & 53,5 & 86,2 & 41,9 & 70,8 & 43,2 & 80,7 \\
\hline & & Miúdo & 41,6 & 11,8 & 52,5 & 26,5 & 39,6 & 15,0 \\
\hline & & Finos & 4,9 & 2,1 & 5,5 & 2,8 & 17,3 & 4,2 \\
\hline \multirow{12}{*}{ 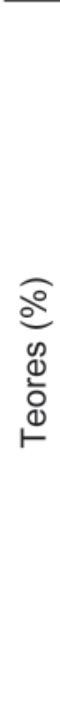 } & \multirow{3}{*}{$\frac{0}{\infty}$} & Graúdo & 65,7 & 66,4 & 67,1 & 65,8 & 70,7 & 66,7 \\
\hline & & Miúdo & 69,5 & 71,2 & 69,3 & 67,2 & 80,6 & 74,0 \\
\hline & & Finos & 47,8 & 49,8 & 48,6 & 46,0 & 51,8 & 53,9 \\
\hline & \multirow{3}{*}{ 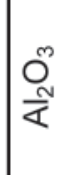 } & Graúdo & 9,2 & 8,7 & 10,5 & 8,8 & 10,3 & 10,7 \\
\hline & & Miúdo & 7,6 & 6,0 & 8,4 & 7,6 & 6,2 & 7,7 \\
\hline & & Finos & 11,1 & 8,4 & 13,5 & 8,8 & 18,0 & 13,1 \\
\hline & \multirow{3}{*}{ O্ঠ } & Graúdo & 9,0 & 8,9 & 6,8 & 9,4 & 0,9 & 1,4 \\
\hline & & Miúdo & 7,6 & 8,5 & 6,5 & 8,4 & 0,5 & 0,9 \\
\hline & & Finos & 13,5 & 17,5 & 10,2 & 17,5 & 1,3 & 1,7 \\
\hline & \multirow{3}{*}{$\frac{u}{\alpha}$} & Graúdo & 7,5 & 7,8 & 5,6 & 7,7 & 6,8 & 6,3 \\
\hline & & Miúdo & 8,4 & 8,2 & 7,9 & 8,7 & 4,3 & 5,5 \\
\hline & & Finos & 20,0 & 16,1 & 19,1 & 19,6 & 14,0 & 13,2 \\
\hline
\end{tabular}

PF = Perda ao Fogo a $1.050^{\circ} \mathrm{C}$, Graúdo -25,4+4,8mm, Miúdo -4,8+0,15mm, Finos -0,15mm

\subsubsection{Influência da natureza e origem}

A composição química do agregado de RCD cinza (com menor conteúdo de cerâmica vermelha) é bastante semelhante à composição química dos agregados de RCD vermelho (com maior conteúdo de cerâmica vermelha) (Tabela 2 e Figura 5c). Foi observado um discreto aumento no teor relativo de alumina para algumas frações granulométricas dos agregados vermelhos, especialmente os originados de Vinhedo (Tabela 2 e Figura 5d).

\section{Conclusões}

As distribuições granulométricas dos RCDs estudados mostram que é necessário um segundo estágio de britagem para adequação da granulometria para uso de agregados reciclados, principalmente para concretos (dimensão máxima de 25,4 mm). Além disso, ressalta-se que a fração abaixo de 4,8 $\mathrm{mm}$ representa até $50 \%$ em massa de todo RCD, sendo que, hoje, não há aplicações difundidas para essa fração, exceto quando aplicada em base de pavimentação.

A composição química dos RCDs está, intrinsecamente, relacionada à fração granulométrica, sendo que as composições químicas do RCD cinza e vermelho são também similares (RCD de Itaquera). Os teores médios, por fração granulométrica, dos Finos da Britagem Primária (FBP) e do Produto Britado (PB) são muito semelhantes, demonstrando semelhança de composição entre as frações acima e abaixo de $25,4 \mathrm{~mm}$.

A Perda ao Fogo (PF) está relacionada, principalmente, à presença de pasta endurecida de cimento nos agregados (fases portadoras de $\mathrm{CaO}$ ) e nos finos de RCD (filossilicatos). A fração miúda 
Composição química de agregados mistos de resíduos de construção e demolição do Estado de São Paulo

Tabela 2 - Composição granuloquímica dos agregados compostos (FBP+PB).

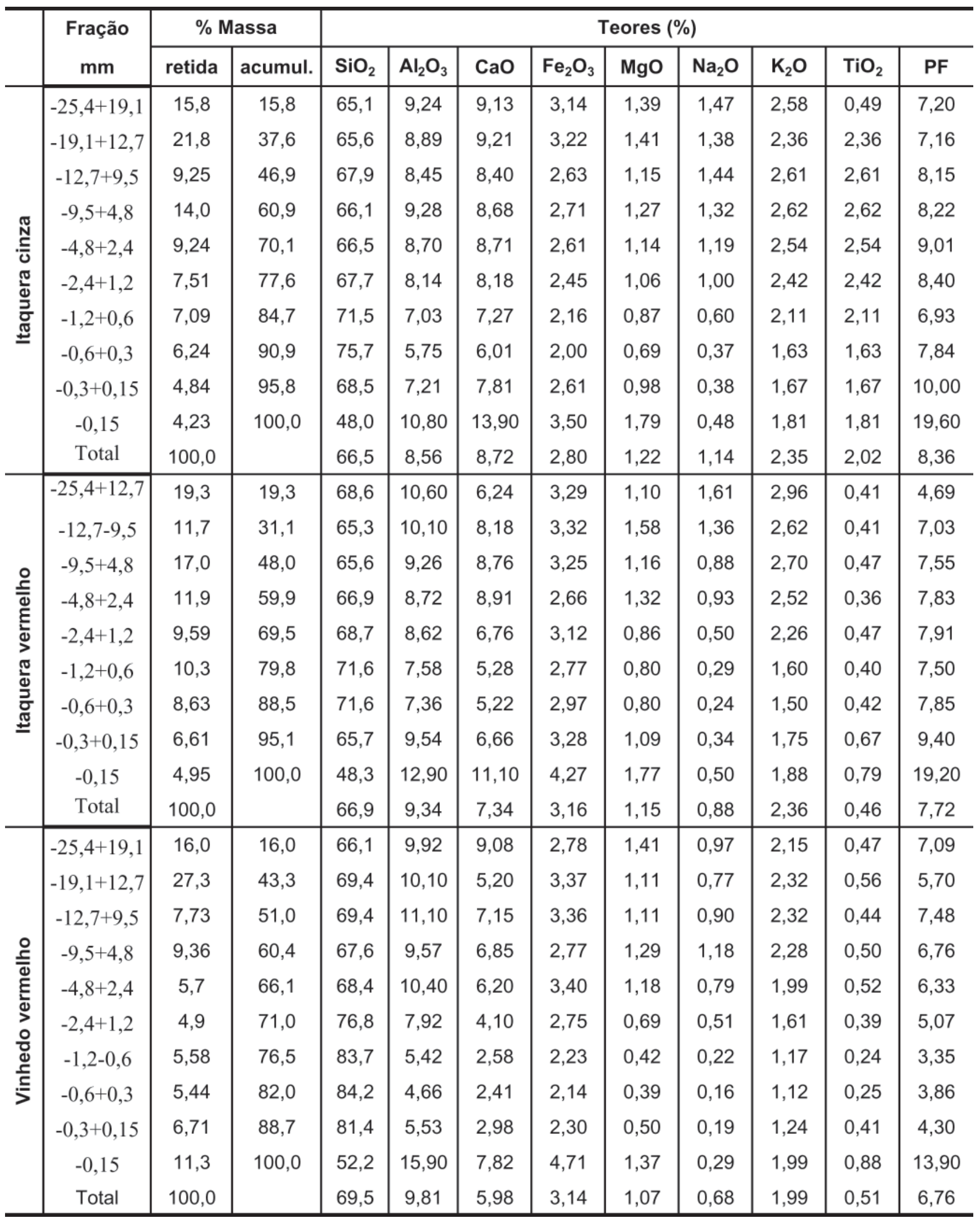

344 REM: R. Esc. Minas, Ouro Preto, 63(2): 339-346, abr. jun. 2010 
Carina Ulsen et al.

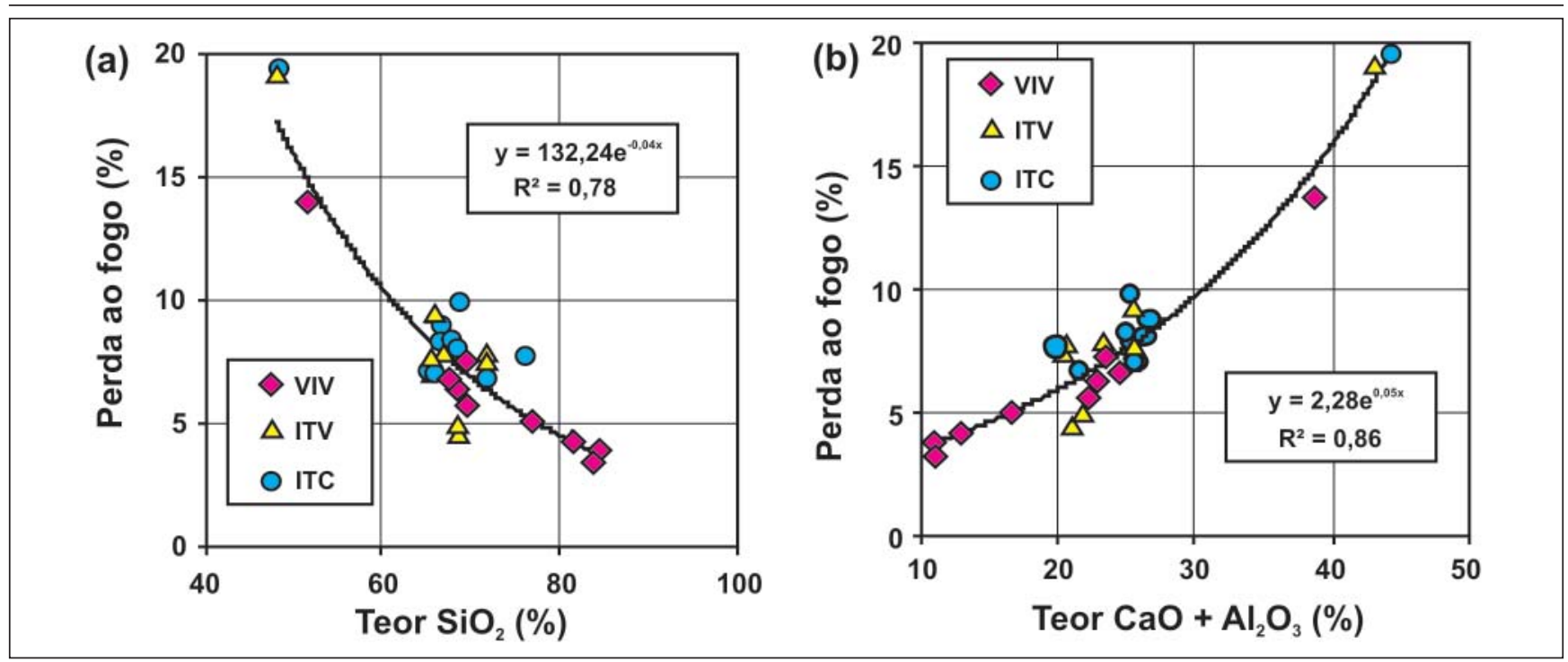

Figura 4 - Relação entre os teores de sílica (a), alumina e óxido de cálcio (b) com a perda ao fogo.

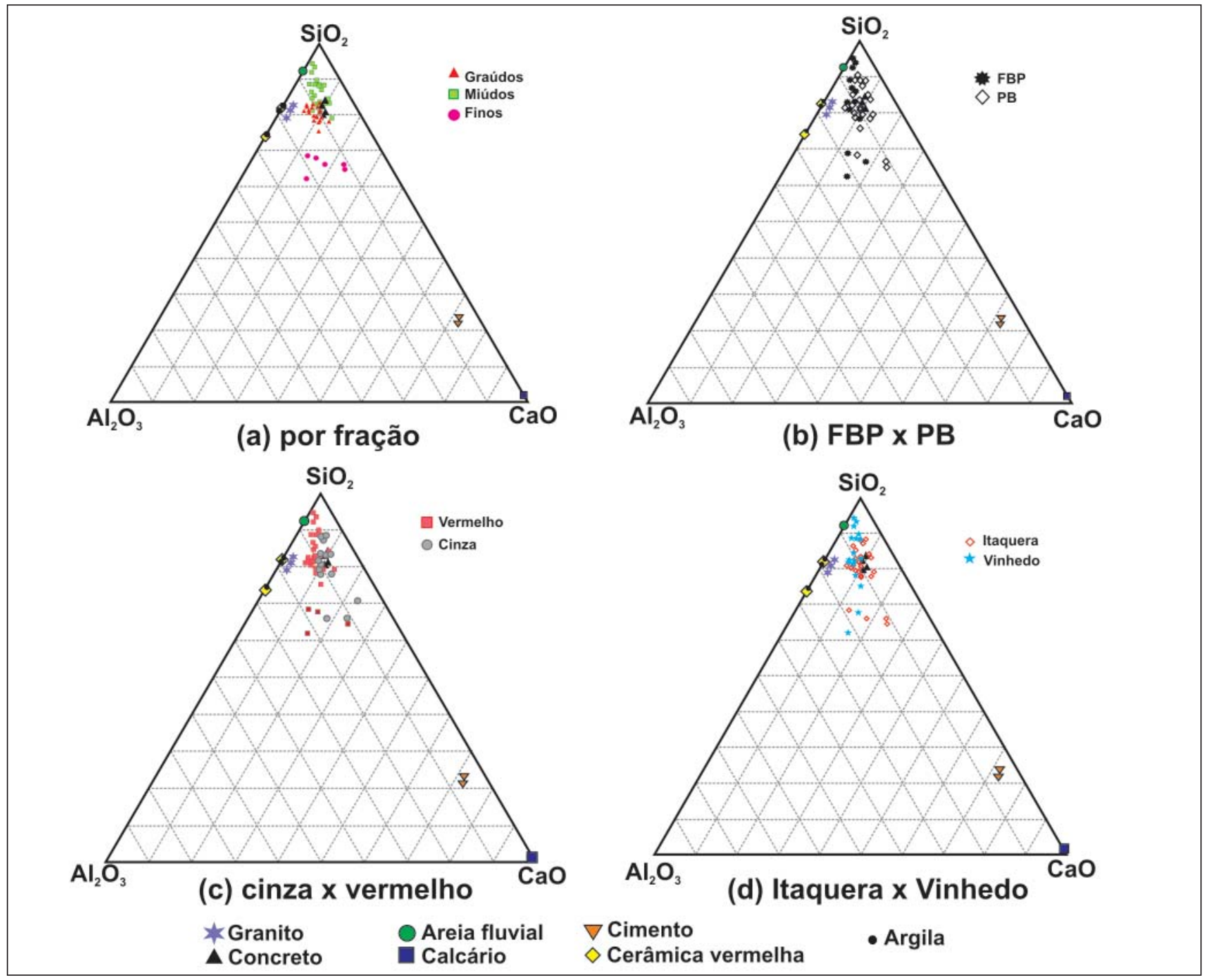

Figura 5 - Composição química dos agregados mistos de RCD segundo o estágio de britagem (a), a classificação visual do resíduo (b), a origem do resíduo (c) e fração granulométrica (d). 
apresenta um enriquecimento nos teores de sílica, indicando liberação da pasta de cimento para os finos $(<0,15 \mathrm{~mm})$. O teor de alumina é influenciado pelo teor de cerâmica vermelha no agregado de RCD e pela contaminação do solo na fração fina $(<0,15 \mathrm{~mm})$. A fração fina pode apresentar um conteúdo de até $50 \%$ de pasta de cimento endurecida e de cerâmica vermelha/argila, indicando uso potencial como matéria-prima alternativa $\left(\mathrm{CaO}\right.$ e $\left.\mathrm{Al}_{2} \mathrm{O}_{3}\right)$ para a indústria do cimento.

\section{Agradecimentos}

À FAPESP pelo financiamento da pesquisa; às administrações das usinas de São Paulo e Vinhedo pela coleta das amostras.

\section{Referências bibliográficas}

ANGULO, S.C., ULSEN, C., KAHN, H., JOHN, V.M. Desenvolvimento de novos mercados para a reciclagem massiva de RCD. In: SEMINÁRIO DE DESEN V O L V I M EN T O SUSTENTÁVEL E A RECICLAGEM NA CONSTRUÇÃO CIVIL, 5. Anais... São Paulo: IBRACON/IPEN, 2002. p. 293-307.

ANGULO, S.C. Caracterização de agregados de resíduos de construção $e$ demolição reciclados e a influência de suas características no comportamento mecânico dos concretos. São Paulo: Escola Politécnica, Universidade de São Paulo, 2005. 149p. (Tese de Doutorado)

ANGULO, S.C., MUELLER, A. Comparative studies about the composition of $C \& D$ recycled aggregates from different sources. In: Recycling 07, 2007. Proceedings... Suiça, 2007.
BIANCHINI, G., MARROCCHINO, E., TASSINARI, R., VACCARO, C. Recycling of construction and demolition waste materials: a chemical and mineralogical appraisal. Waste Management, 25, p. 149-159, 2005.

CARNEIRO, A.P. et al. Caracterização do entulho de Salvador visando à produção de agregado reciclado. In: ENCONTRO NACIONAL DE TECNOLOGIA DO AMBINETE COSNTRUÍDO, 7. Anais... Salvador: ANTAC, 2000.

CONSELHO NACIONAL DO MEIO AMBIENTE (CONAMA). Resolução n 307. Brasília, 2002.

EUROPEAN COMMISSION (EC). Management of construction and demolition waste. 2000. FERRAZ, G.R. et al. Estações de classificação e transbordo na cidade de São Paulo. In: SEMINÁRIO DE DESENVOLVIMENTO SUSTENTÁVEL E A RECICLAGEM NA CONSTRUÇÃO CIVIL, 4. Anais... São Paulo: IBRACON/IPEN, 2001. p.75-86.

HENDRIKS, C.F. The building cycle. Holanda: Aeneas, 2000. 231 p.

JOHN, V.M. Reciclagem de resíduos na construção civil - contribuição à metodologia de pesquisa e desenvolvimento. São Paulo: Escola Politécnica, Universidade de São Paulo, 2000. 102p. (Tese de Livre Docência).

JUNGMANN, A., QUINDT, J. Alljig - technology for separation of building rubble and other secondary raw materials. In: Global Symposium on Recycling, Waste Treatment and Clean Technology, 1999. San Sebastian. Proceedings... Warrendale: TMS/NASMET, 1999. p. 367-379.

LIMBACHIYA. M. C., MARROCCHINO, E., KOULORIS, A. Chemical-mineralogical characterisation of coarse recycled concrete agreggate. Waste Management, v. 27, p. 210-208, 2006.

LINSZ, E., MUELLER, A. High-performance sonic impulses: an alternative method for processing of concrete. International Journal of Mineral Processing, v.74, p. 199-218, 2004.

MUELLER, A. Recycling von Mauerwerkbruch: stand und neue verwertungswege (teil 1). Ziegelindustrie International, v. 56, p. 17-25, 2003.

NAGATAKI, S., GOKCE, A., SAEKI, T., HISADA, M. Assessment of recycling process induced damage sensitivity of recycled concrete aggregates. Cement and Concrete Research, v. 34, p. 965-971, 2004.

ULSEN, C. Caracterização tecnológica de resíduos de construção e demolição. São Paulo: Escola Politécnica, Universidade de São Paulo, 2006. 20p. (Dissertação de Mestrado).

PETERSEN, I. F., MINKKINEN, P., ESBENSEN, K.H. Representative sampling for reliable data analysis: Theory of Sampling. Chemometrics and Intelligent Laboratory Systems, v. 77, p. 261-277, 2005.

PINTO, T.P. Metodologia para a gestão diferenciada de resíduos sólidos da construção urbana. São Paulo: Escola Politécnica, Universidade de São Paulo, 1999. 189p. (Tese de Doutorado).

RILEM RECOMMENDATION. Specification for concrete with recycled aggregates. Materials and Structures, v. 27, p. 557-59, 1994.

SCHNEIDER, D. M. Transporte de resíduos de construção e demolição na cidade de São Paulo. São Paulo: Faculdade de Saúde Pública, Universidade de São Paulo, 2003. 103p. Dissertação de Mestrado).

Artigo recebido em 16/10/2008 e aprovado em 15/10/2009.

\section{A REM tem novo endereço: FUNDAÇÃO GORCEIX - REM Rua Carlos Walter Marinho Campos, 57 - Vila Itacolomy 35400-000 - Ouro Preto - MG www.rem.com.br}

